\title{
Psicooncología
}

ISSN: 1696-7240

http://dx.doi.org/10.5209/PSIC.63648

\section{Validación de la Escala de Ansiedad Preoperatoria y de Información Ámsterdam (APAIS) en mujeres latinoamericanas con cáncer de mama: Estudio México - Costa Rica}

Karla Janeth Méndez-Meneses; María Luisa Rebolledo García²; Stephanie Díaz Chacón²; Luis Alberto Rodríguez Vázquez ${ }^{3}$; Brenda Lizeth Acosta Maldonado ${ }^{4}$; Blanca MantillaMaya $^{5}$; Eva González-Rodríguez ${ }^{6}$; José Luis Aguilar Ponce ${ }^{7}$; Oscar Galindo Vázquez; Liliana Rivera-Fong ${ }^{9 *}$

Recibido: el 17 de diciembre de 2018 / Aceptado el 7 de marzo de 2019

Resumen: El tratamiento más frecuente contra el cáncer es la cirugía; sin embargo es un procedimiento invasivo que se ha asociado con la presencia de sintomatología psicológica hasta en el $76 \%$ de los pacientes. El estudio de la ansiedad ante la cirugía requiere de herramientas específicas adaptadas a las peculiaridades del constructo; entre ellas se encuentra la Escala de Ansiedad Preoperatoria y de Información Ámsterdam (APAIS) que ha sido validada en pacientes sometidos a diversas cirugías en múltiples países; mostrando consistentemente propiedades psicométricas adecuadas. Objetivo: Obtener la confiabilidad y validez de la APAIS en población oncológica para población latinoamericana. Método: Estudio transversal, no experimental, de análisis psicométrico que incluyó una muestra no probabilística de 117 mujeres con edades entre 25 y 75 años, todas diagnosticadas con cáncer de mama y programadas para cirugía en un hospital público de México (57 mujeres) o Costa Rica (63 mujeres). Resultados: Los seis reactivos de la Escala APAIS mostraron adecuada distribución de las contestaciones de las participantes entre las opciones de respuestas, tener capacidad de discriminación entre grupos extremos y contribuyeron a la consistencia interna del instrumento. El análisis factorial de máxima verosimilitud con rotación varimax mostró una estructura que explicó el 58,58\% de la varianza

1 Universidad Latina, Campus Sur. México. Instituto Nacional de Cancerología, México

E-mail: liebe_janeth21@hotmail.com.

2 Hospital México. Caja Costarricense de Seguro Social. Costa Rica.

E-mail: mlreboll@ccss.sa.cr

3 Universidad Latina, Campus Sur. México. Instituto Nacional de Cancerología, México.

E-mail: vonska@hotmail.com

4 Instituto Nacional de Cancerología, México.

E-mail: brenda_ao@hotmail.com

5 Universidad Latina, Campus Sur. México. Secretaría de Salud. México.

E-mail: psicbmm@gmail.com

6 Universidad Latina, Campus Sur. México. evagonzalez69@hotmail.com.

7 Instituto Nacional de Cancerología, México.

E-mail: joluagpo@hotmail.com

8 Universidad Latina, Campus Sur. México.Instituto Nacional de Cancerología, México. psigalindo@yahoo.com

9 Universidad Latina, Campus Sur. México.Universidad Nacional Autónoma de México Instituto Nacional de Cancerología, México.

E-mail:1meylenf@hotmail.com

* Dirección de correspondencia: Mtra. Liliana Rivera Fong. Universidad Latina, Campus Sur. México. C/ Pedro Henríquez Ureña 173, Col. Los Reyes. Del Coyoacán, México, CDMX. E-mail: 1meylenf@hotmail.com 
con dos factores, la cual fue confirmada por análisis factorial confirmatorio; la escala total mostró una consistencia interna de $\alpha=0,816$. Dicha escala mostró adecuada sensibilidad y especificidad con un punto de corte de 14 puntos. Conclusiones: La escala APAIS muestra propiedades psicométricas adecuadas para considerarla válida y confiable para evaluar ansiedad prequirúrgica en mujeres con cáncer de mama.

Palabras Claves: cáncer, cirugía, APAIS, ansiedad prequirúrgica, validez.

\title{
[en] Validation of the Amsterdam Pre-surgery Anxiety and Information
} Scale in Latino American women with breast cancer: Mexico - Costa Rica research

\begin{abstract}
Surgery is the most frequent and efficient treatment for cancer, however, it is an invasive procedure that has been associated with psychological symptoms in up to $76 \%$ of patients. Health professional require specific and adapted tools to evaluate anxiety related to surgery, but there are few instruments to evaluate it. An option is the Amsterdam Preoperative Anxiety and Information Scale (APAIS), which has been validated in multiple countries with patients undergoing different surgeries. Validations have shown APAIS has adequate psychometric properties. Aim: To obtain the reliability and validity of APAIS in the oncology population for the Latin American population. Method: Crosssectional, non-experimental, psychometric analysis study that included a non-probabilistic sample of 117 women aged between 25 and 75 years, all patients have been diagnosed with breast cancer and have been scheduled for surgery in a public hospital in Mexico (57 women) or Costa Rica (63 women). Results: The six items of the APAIS Scale showed an adequate distribution of the responses of the participants among the response options, the ability to discriminate between extreme groups and contributed to the internal consistency of the instrument. The factor analysis of maximum likelihood with varimax rotation showed a structure that explained $58.58 \%$ of the variance with two factors, this structure was confirmed by a confirmatory factor analysis. The total scale showed an internal consistency of $\alpha=.816$. This scale showed adequate sensitivity and specificity with a cut-off point of 14 points. Conclusions: The APAIS scale shows adequate psychometric properties to be considered valid and reliable to evaluate pre-surgical anxiety in patients with cancer.
\end{abstract}

Keywords: neoplasm, surgery, APAIS, anxiety related to surgery, validity.

Sumario. 1. Introducción 2. Método 3. Resultados 4. Discusión y conclusiones 5. Referencias bibliográficas.

Cómo citar: Méndez-Meneses KJ, Rebolledo García ML, Díaz Chacón S, Rodríguez Vázquez LA, Acosta Maldonado BL; Mantilla-Maya B, González-Rodríguez E, Aguilar Ponce JL, Galindo Vázquez O, Rivera-Fong L. Validación de la Escala de Ansiedad Peroperatoria y de Información Ámsterdam (APAIS) en mujeres latinoamericanas con cáncer de mama: Estudio México - Costa Rica. Psicooncología 2019; 16(1): 73-88. doi: 10.5209/PSIC.63648.

\section{Introducción}

A nivel mundial, el cáncer de mama representa más del 10\% de todos los casos nuevos de cáncer en el mundo y constituye más del $1.6 \%$ de todas las muertes de mujeres en el mundo ${ }^{(1)}$. La epidemiología en México indica que se tiene una incidencia de 20.044 casos; una prevalencia de 75.529 personas viviendo con cáncer de mama y una mortalidad anual de 5680 , siendo ésta la principal causa de morbilidad en mujeres de 20 a 50 años $^{(2,3)}$. Por otra parte, en Costa Rica, el GLOBOCAN reporta cifras de una incidencia de 1.145 casos por cada 100.000 habitantes; una prevalencia 
4.118 personas viviendo con cáncer por cada 100.000 habitantes y una mortalidad de 363 por cada 100.000 habitantes por esta causa ${ }^{(4)}$.

El abordaje médico de las pacientes con cáncer de mama puede implicar múltiples procedimientos o estrategias terapéuticas como la quimioterapia, radioterapia, tratamiento hormonal y/o cirugía; esta última modalidad de tratamiento puede ser empleada en diversas fases de la enfermedad al tener entre sus objetivos el diagnóstico, la curación del cáncer en tanto sea posible, extirpación del tumor ya sea de forma total o parcial, detectar el avance de los ganglios linfáticos, restaurar la forma del seno después de extraer el tumor o aliviar los síntomas del cáncer avanzado ${ }^{(5,6)}$, por lo que una paciente puede enfrentarse a más de una cirugía a lo largo del proceso de atención $^{(7)}$.

Hasta el momento se ha identificado que, a pesar de la importancia y avance de otros tratamientos; la cirugía es el tratamiento que proporciona un número superior de beneficios para el tratamiento de cáncer de mama, al brindar las mayores probabilidades de curación ${ }^{(7)}$; sin embargo, también puede ocasionar una serie de reacciones y manifestaciones psicológicas como ansiedad que, ante un procedimiento invasivo como la cirugía, puede adquirir ciertas particularidades en su presentación como que el contenido de las preocupaciones tiende a centrarse en la anestesia y/o el procedimiento quirúrgico en particular ${ }^{(8)}$, miedo al dolor durante y post-quirúrgico, sentimiento de pérdida del control corporal, la posibilidad de presentar cicatrices en el cuerpo y, en casos de cirugías mayores, presencia de pensamientos de muerte asociados al procedimiento y/o la anestesia ${ }^{(7,8)}$.

Los hallazgos actuales indican que el riesgo de presentar ansiedad asociada a una cirugía es significativamente mayor antes del procedimiento con respecto a otros momentos o situaciones particulares del propio tratamiento oncológico. Al respecto se estima que al enfrentarse a una enfermedad oncológica aproximadamente el $24 \%$ de los pacientes presentan algún trastorno mental ${ }^{(9)}$ y entre el $25 \%$ y $50 \%$ de los pacientes experimentan niveles elevados de malestar emocional; siendo ansiedad, depresión y trastornos del sueño los más referidos por los pacientes ${ }^{(10)} \mathrm{y}$, de manera específica, al valorar ansiedad se ha observado que, dependiendo de la metodología de evaluación, entre el $0,9 \%$ y el $49 \%$ de los pacientes reportan sintomatología de ansiedad debido principalmente al hecho de que tiene una enfermedad o dolencia que impacta negativamente en su funcionamiento ${ }^{(11,12)}$. Sin embargo, este porcentaje puede ser incluso mayor al ofrecerles un procedimiento quirúrgico, momento en el que hasta el $76 \%$ de los pacientes oncológicos presenten sintomatología de ansiedad $^{(13)}$; situación a la que se les suma una serie de reacciones emocionales como inseguridad, miedo, nerviosismo y estrés ${ }^{(14)}$.

Probablemente debido a su alta incidencia, es frecuente que la ansiedad prequirúrgica sea considerada por el personal de salud como una respuesta esperada o "normal"; concepción que le confiere características de un estado transitorio y que no requeriría atención especializada; sin embargo se tiene documentado que, en el caso de pacientes oncológicos, la presencia de niveles altos de ansiedad prequirúrgica puede influir de manera negativa en la evolución de paciente tanto en la toma de decisión de someterse al procedimiento, como en el tiempo requerido de hospitalización, uso de medicamentos e incremento de riesgo de desarrollar complicaciones infecciosas o baja adherencia a indicaciones médicas ${ }^{(13,15-18)}$. De manera que se convierte en una entidad con impacto significativo en la atención del paciente oncológico y debe ser abordada de manera estrecha. 
Adicionalmente, se ha identificado que existen factores de riesgo para que un paciente presente sintomatología de ansiedad previa a la cirugía en niveles altos o ésta pueda prolongarse después del procedimiento. Para su mejor comprensión, dichos factores han sido clasificados con base en su origen y se dividen en: (a) elementos relacionados con la anestesia; como el tipo de fármacos que se empleará, la modalidad de la anestesia, las asociaciones que el paciente haga sobre la anestesia y la gravedad o posibles complicaciones, experiencias negativas previas con la anestesia, miedo a que durante el procedimiento disminuya o se pierda el efecto farmacológico y derive en permanecer despierto durante el acto quirúrgico y despertar con secuelas debido a una mala administración anestésica ${ }^{(19,20)}$; (b) variables asociadas al procedimiento propiamente como preocupaciones sobre la separación de la familia por la hospitalización, falta o pérdida de autonomía, las condiciones físicas al momento del procedimiento, posibles implicaciones en funcionalidad o estética derivados de la cirugía, la supervivencia y el tiempo y complicaciones asociadas a la recuperación ${ }^{(21,22)}$. En este sentido, se ha recomendado que el personal de salud brinde al paciente información precisa y adaptada a su situación clínica para disminuir los niveles de ansiedad asociada a procesos cognitivos disfuncionales derivados de falta de información o imprecisión en la misma ${ }^{(23)}$.

En relación a los momentos claves para la evaluación de la ansiedad relacionada con la cirugía (ARC); se ha descrito que tiene mayor riesgo de iniciar su desarrollo una semana antes del acto quirúrgico e intensificarse en las horas previas del ingreso hospitalario; éste último momento de evaluación tiene una correlación positiva con las complicaciones post-quirúrgicas, el tiempo de convalecencia, la percepción del dolor a las 24, 48 y 72 horas postoperatorias y el bienestar de los pacientes sometidos al tratamiento ${ }^{(24)}$.

Para su evaluación existe la "Amsterdam Preoperative Anxiety and Information Scale (APAIS)" que cuenta con seis reactivos en formato de respuesta tipo Likert que miden miedo a la anestesia $[\alpha=, 86]$, miedo al procedimiento $[\alpha=, 86]$ y necesidad de información $[\alpha=, 68]$ ) con una varianza explicada del $72 \%$; con un punto de corte de 11 puntos para considerar que un paciente requiere de atención especializada ${ }^{(29)}$. Esta escala ha sido validada en múltiples muestras de pacientes de cirugía general a lo largo del mundo ${ }^{(30,35-37)}$ demostrando su utilidad; sin embargo no se ha validado en población oncológica.

Es claro que la presencia de ansiedad pre-quirúrgica en pacientes oncológicos tiene gran impacto tanto en el procedimiento mismo como en la evolución de paciente y el requerimiento de los servicios de salud; sin embargo su estudio ha sido limitado y para ello la validación de instrumentos especializados sería de gran ayuda; al respecto, la APAIS ha demostrado ser una herramienta eficaz para el tamizaje de ansiedad en pacientes pre-quirúrgicos y ofrece amplias ventajas sobre otros instrumentos similares al omitir reactivos relacionados con sintomatología fisiológica o afectiva que pudieran ser confusos en pacientes con cáncer ${ }^{(30)}$. Es por ello que el presente estudio se planteó como objetivo evaluar las propiedades psicométricas de la Escala de Ansiedad Preoperatoria y de Información de Ámsterdam (APAIS) en mujeres con cáncer de mama. 


\section{Método}

Para el proceso de validación de la escala APAIS se incluyeron 117 mujeres con cáncer de mama [63(53,85\%) costarricenses y 54(46,15\%) mexicanas]. El cálculo de la muestra estuvo basado en el criterio de Nunnally ${ }^{(31)}$ que consideran la inclusión de por lo menos diez participantes por ítem; considerando que el instrumento tiene seis reactivos, se requería de un mínimo de 60 participantes; por lo que se incluyó un estimado de estos participantes por país.

Para la incorporación de participantes se consideró que fueran pacientes mayores de edad con diagnóstico confirmado de cáncer de mama, que estuvieran recibiendo atención médica en un hospital público de estos países; que tuvieran programado una cirugía mayor en el transcurso del siguiente mes a la aplicación, que comprendieran el idioma español, que supieran leer y escribir y que aceptaran participar de manera voluntaria.

Los participantes contestaron de manera concurrente la APAIS y la escala de ansiedad y depresión hospitalaria (HADS). La escala APAIS consta de seis reactivos con formato de respuesta de escala Likert de cinco punto (1 - 5) en la que uno significa "Nada" y cinco "Muchísimo"; para fines de este estudio se tomó como base la escala original y fue sometida a un proceso de traducción - retraducción. En la primera fase, un psicólogo hispanohablante con dominio del idioma inglés realizó la traducción de la escala original; la versión en español fue proporcionada a un psicólogo anglosajón con dominio del idioma español para su traducción al idioma inglés nuevamente. La versión original fue contrastada con la versión proporcionada por el psicólogo anglosajón para asegurar la integridad en la intensión de cada reactivo.

Con el objetivo de asegurar la comprensión de cada uno de los reactivos por parte de la de la población blanco; la versión obtenida a partir de la traducción retraducción fue aplicada a 15 mujeres con cáncer de mama que tuvieran programada durante la siguiente semana una cirugía que implicara anestesia general. En esta fase, cada una de las mujeres contestaron el instrumento y posterior a ello se les preguntó sobre la existencia de palabras complejas, uso de frases que pudieran ser ofensivas o redacción de difícil comprensión en cada uno de los reactivos; la información brindada por las participantes fue capturada y se consideró que un reactivo requeriría ajustes en su redacción cuando presentara dificultades para ser comprendido por el $20 \%$ o más de las participantes.

La escala HADS cuenta con 14 reactivos en su versión original (siete para evaluar ansiedad y siete para depresión; todos con una escala Likert de cuatro puntos que van de cero a tres, cada reactivo cuenta con opciones de respuesta diferentes)(27,34). Además se tomaron del expediente de las paciente los principales datos clínicos y sociodemográficos. Los datos de ambos países fueron capturados en una base de datos que fue analizada en el programa estadístico SPSS IBM v22.

Como parte del análisis estadístico de la APAIS se obtuvieron las medidas de tendencia central (media, mediana y moda) de cada reactivo con el fin de conocer la distribución de las respuestas entre las opciones de respuestas y se calculó la asimetría y curtosis de cada reactivo; considerando como parámetros esperados de normalidad puntajes $>-2$ y $<2$ en ambos estadísticos. Posteriormente se calificó la escala sumando el total de reactivos y se obtuvieron estadísticos de tendencia central y dispersión de dicho puntaje; con base en ello se establecieron dos grupos extremos (con los sujetos del cuartil 25 y 75). Con el objetivo de conocer la capacidad de 
discriminación de cada reactivo, se llevó a cabo la prueba de Kolmogorov-Smirnov para determinar el tipo de análisis más indicado para hacer una comparación entre grupos independientes con cada reactivo, en dicha prueba aquellos reactivos que no mostraran diferencias estadísticamente significativas entre grupos $(p>0,05)$ fueron eliminados de la escala y de los siguientes análisis. Posterior a ello se evaluó la contribución de cada reactivo a la consistencia interna del reactivo mediante el Alfa de Cronbach total del instrumento y la reportada en caso de que se eliminara cada uno de los reactivos; se consideró descartar aquellos reactivos que al ser eliminados incrementan en más de 0,20 la consistencia interna del instrumento.

Con los reactivos que discriminaron se realizó un análisis de tablas cruzadas para conocer la correlación entre ítems y determinar el tipo de rotación a empelar en el análisis factorial de máxima verosimilitud (varimax en caso de reactivos independientes y oblimin en caso de reactivos con alta correlación). Durante el análisis factorial exploratorio se determinó como criterio para mantener los reactivos que mostraran una carga factorial $>0,40$ en un factor, mostrar agrupaciones con valores Eigen superiores a 1 y que contribuyeran en por lo menos $10 \%$ a la varianza explicada; las agrupaciones de tres o más reactivos fueron denominadas factores y agrupaciones de dos reactivos o reactivos independientes se nombraron indicadores.

Adicionalmente se llevó a cabo un análisis factorial confirmatorio con el objetivo de evaluar empíricamente la estructura observada en el análisis factorial exploratorio. En dicho análisis se empleó un método de máxima verosimilitud en que se fijaron las cargas de varianza de las variables latentes en 1.0 y los términos de la varianza de error se determinaron como parámetros libres. Se consideró que un modelo tenía buen ajuste cuando (a) el residuo cuadrático medio estandarizado (SMRM) $<0,80$, (b) el error medio cuadrático de aproximación (RMSEA) $<0,06$ y (c) el índice de comparación de ajuste (CFI) y el índice de Tucker Lewis (TLI) estuvieron entre 0,90 y 0,95 . Un modelo tiene un ajuste adecuado cuando (a) RMSEA $<0,80$ o (b) CFI y TLI estuvieron cercanos a 0,90 . Un modelo presenta poco ajuste cuando (a) RMSEA se encuentre entre 0,80 y 0,10 o (b) CFI y TLI estén alejados de 0,90 .

Una vez establecida la estructura factorial de la escala, se estimó la consistencia interna tanto de la escala total como de cada uno de los factores o indicadores mediante el Alfa de Cronbach. Complementariamente, se obtuvo la validez concurrente de la APAIS mediante un análisis de correlación con $r$ de Pearson entre el APAIS (escala total y cada uno de los factores) y la subescala de ansiedad del HADS; se examinó la validez discriminante mediante un análisis de correlación $r$ de Pearson entre el APAIS (escala total y cada uno de los factores) y la subescala de depresión del HADS.

Finalmente, con base en la revisión llevada a cabo por Terol-Cantero y colaboradores en $2015^{(38)}$, se consideró el punto de corte del HADS ansiedad (8 puntos) para determinar a los pacientes con sintomatología clínica significativa; puntaje que se encuentra por debajo del punto de corte para casos clínicos, pero incluye a población subclínica que requieren evaluación para descartar la presencia de un trastorno. Con base en ello se evaluó su sensibilidad, especificidad y valores predictivos negativos y positivo de la escala de la escala APAIS con diversos puntajes para determinar el de mejor ajuste. 


\section{Participantes}

En la fase de traducción y adaptación lingüística se incluyeron 15 mujeres con cáncer de mama que tuvieron una edad promedio de 40 años; de las cuales $8(53,3 \%)$ reportaron ser solteras, $6(40 \%)$ casadas y $1(6,7 \%)$ separada; en relación al nivel educativo, $1(6,7 \%)$ participante reportó no contar con educación formal, $4(26,7 \%)$ contaba con educación secundaria, 7(46,7\%) con formación de nivel superior y 3 (20\%) con formación universitaria.

Todas las participantes tenían programada dentro de las próximas dos semanas a su evaluación una cirugía que implicaba anestesia general; de ellas $9(60 \%)$ se someterían por primera vez a un procedimiento quirúrgico. En relación a las cirugías programadas $8(53,3 \%)$ se someterían a una mastectomía, $4(26,7 \%)$ a una segmentectomía y $3(20 \%)$ a una reconstrucción mamaria.

En la fase de validación del APAIS se incluyeron 117 mujeres con cáncer de mama [63 (53,85\%) costarricenses y $54(46,15 \%)$ mexicanas] que tenían programada una cirugía relacionada con el tratamiento oncológico durante el próximo mes y cuyas características sociodemográficas se describen en la tabla 1; mientras que las características clínicas se describen en la tabla 2, en la cual es importante resaltar que en la sección de comorbilidades algunas participantes presentaban más de una enfermedad crónicas.

Tabla 1. Características sociodemográficas de las participantes $(n=117)$

\begin{tabular}{lccc}
\hline & Mexicanas $(\mathrm{n}=54)$ & Costarricenses $(\mathrm{n}=63)$ & Total $(\mathrm{n}=117)$ \\
\hline Edad & & & \\
Media (d.e.) & $47,17(10,19)$ & $58,11(13,12)$ & $53(13,02)$ \\
Mediana (rango) & $45(25-71)$ & $58(27-85)$ & $51(25-85)$ \\
Estado civil & $f(\%)$ & $f(\%)$ & $f(\%)$ \\
Soltera & $22(40,7)$ & $14(22,2)$ & $36(30,8)$ \\
Casada & $24(44,4)$ & $36(57,1)$ & $60(51,3)$ \\
Divorciada & $8(14,8)$ & $4(6,3)$ & $12(10,3)$ \\
Viuda & $0(0,0)$ & $9(14,3)$ & $9(7,7)$ \\
Nivel de estudios & $f(\%)$ & $f(\%)$ & $f(\%)$ \\
Ninguna & $1(1,9)$ & $6(9,5)$ & $7(6,0)$ \\
Primaria & $7(13,0)$ & $27(42,9)$ & $34(29,1)$ \\
Secundaria & $11(20,4)$ & $12(19,0)$ & $23(19,7)$ \\
Medio superior & $19(35,2)$ & $10(15,9)$ & $29(24,8)$ \\
Licenciatura & $14(25,9)$ & $6(9,5)$ & $20(17,1)$ \\
Posgrado & $2(3,7)$ & $2(3,2)$ & $4(3,4)$ \\
\hline
\end{tabular}


Tabla 2. Características clínicas de las participantes $(\mathrm{n}=117)$

\begin{tabular}{|c|c|c|c|}
\hline & $\begin{array}{l}\text { Mexicanas } \\
(n=54)\end{array}$ & $\begin{array}{c}\text { Costarricenses } \\
\qquad(\mathrm{n}=63)\end{array}$ & $\begin{array}{c}\text { Total } \\
(\mathrm{n}=117)\end{array}$ \\
\hline Estadio clínico & $f(\%)$ & $f(\%)$ & $f(\%)$ \\
\hline I & $9(16,7)$ & $32(50,8)$ & $41(35,0)$ \\
\hline II & $36(66,7)$ & $26(41,3)$ & $62(53,0)$ \\
\hline III & $8(14,8)$ & $5(7,9)$ & $13(11,1)$ \\
\hline IV & $1(1,9)$ & $0(0,0)$ & $1(0,9)$ \\
\hline Comorbilidades & $f(\%)$ & $f(\%)$ & $f(\%)$ \\
\hline Diabetes & $8(14,8)$ & $17(27,0)$ & $25(21,4)$ \\
\hline Hipertensión & $5(9,3)$ & $32(50,8)$ & $37(31,6)$ \\
\hline Asma & $3(5,6)$ & $4(6,3)$ & $7(6,0)$ \\
\hline Osteoporosis & $1(1,9)$ & $2(3,2)$ & $3(2,6)$ \\
\hline Otras* & $10(18,5)$ & $15(23,8)$ & $25(21,4)$ \\
\hline Ninguna & $25(46,3)$ & $17(27,0)$ & $42(35,9)$ \\
\hline Tratamientos adicionales a la cirugía & $f(\%)$ & $f(\%)$ & $f(\%)$ \\
\hline Ninguno & $5(9,3)$ & $3(4,8)$ & $8(6,8)$ \\
\hline Quimioterapia & $6(11,1)$ & $3(4,8)$ & $9(7,7)$ \\
\hline Radioterapia & $5(9,3)$ & $6(9,5)$ & $11(9,4)$ \\
\hline Terapia hormonal & $9(16,7)$ & $13(20,7)$ & $22(18,8)$ \\
\hline Quimioterapia + Radioterapia & $11(20,4)$ & $5(7,9)$ & $16(13,7)$ \\
\hline Quimioterapia + Terapia hormonal & $1(1,9)$ & $1(1,6)$ & $2(1,7)$ \\
\hline Radioterapia + Terapia hormonal & $17(31,5)$ & $32(50,8)$ & $49(41,9)$ \\
\hline \multicolumn{4}{|l|}{ Tipo de cirugía } \\
\hline Mastectomía radical & $30(55,6)$ & $31(49,2)$ & $61(53,5)$ \\
\hline Tumorectomía & $1(1,9)$ & $5(7,9)$ & $6(5,1)$ \\
\hline Segmentectomía & $12(22,2)$ & $18(28,6)$ & $30(25,6)$ \\
\hline Cuadrantectomía & $3(5,6)$ & $8(12,7)$ & $11(9,4)$ \\
\hline Reconstitución mamaria & $8(14,9)$ & $1(1,6)$ & $9(7,7)$ \\
\hline
\end{tabular}

*Se incluyeron enfermedades como hipotiroidismo, hipertiroidismo, gastritis crónica, lupus y arritmia cardiaca. 


\section{Resultados}

En la fase de traducción y adaptación lingüística, todos los reactivos fueron reportados como claros y fáciles de comprender por los participantes; así como reportaron que ninguno empleaba palabras complejas o difíciles de interpretar. Debido a esto no se realizaron ajustes a la redacción de los reactivos.

En la fase de validación del instrumento, los análisis descriptivos mostraron que el total de reactivos del APAIS tenían adecuada distribución entre las opciones de respuesta debido a que ninguna opción de respuesta concentró más $50 \%$ de las respuestas de los participantes. Adicionalmente, todos los reactivos mostraron adecuadas condiciones de asimetría y curtosis $(<2)$; lo que indicó que los participantes no respondieron con una tendencia a alguno de los extremos, ni se concentraron en un número limitado de opciones de respuesta.

Posteriormente se calificó el instrumento con el total de reactivos de la escala APAIS. El puntaje promedio de los participantes fue de 17,75 con una desviación estándar de 6,32, donde el puntaje máximo del cuartil inferior fue 12,50 y el puntaje mínimo del cuartil superior fue de 23; a partir de lo cual se formaron los grupos extremos.

Debido a que el puntaje total del APAIS y de cada uno de los reactivos no mostraron una distribución normal en la prueba de Kolmogorov-Smirnov $(p>0,05)$ se llevaron a cabo pruebas de comparación no paramétrica entre los grupos extremos de la muestra (cuartil inferior vs cuartil superior) mediante U de Mann Whitney en la que se observó que todos los reactivos mostraron capacidad de discriminación al identificar diferencias entre grupos extremos, por lo que todos fueron conservados. Posteriormente, el Alfa de Cronbach indicó una adecuada consistencia interna hasta el momento $(\alpha=0,816)$; la cual disminuía al eliminar cualquier reactivo, por lo que todos fueron conservados (Tabla 3 ).

Tabla 3. Análisis parcial de confiabilidad

\begin{tabular}{lc}
\hline Reactivo & $\begin{array}{c}\text { Alfa si se elimina } \\
\text { el reactivo }\end{array}$ \\
\hline APAIS 1. Estoy preocupada por la anestesia. &, 774 \\
APAIS 2. La anestesia está en mi mente constantemente. &, 784 \\
APAIS 3. Me gustaría saber lo más posible sobre la anestesia. &, 798 \\
APAIS 4. Estoy preocupada por el procedimiento &, 774 \\
APAIS 5. El procedimiento está en mi mente constantemente &, 785 \\
APAIS 6. Me gustaría saber lo más posible sobre el procedimiento &, 806 \\
\hline
\end{tabular}

Como parte del análisis de validez, el análisis de matrices cruzadas determinó que el $73.33 \%$ de los cruces tuvieron una correlación baja $(r<0,50, p<0,50)$ y el $26,66 \%$ una correlación moderada $(r>0,50 \mathrm{a}<0,80, p<0,50)$. Con base en estos hallazgos se consideró que los reactivos son independientes entre sí y se decidió llevar a cabo un análisis factorial exploratorio por máxima verosimilitud con rotación varimax; 
el cual arrojó un $\mathrm{KMO}=0,679$ y una prueba de esfericidad de Bartlett significativa $\left(\mathrm{x}^{2}=289,58, \mathrm{gl}=15, p<0,001\right)$, los cuales muestran aceptable adecuación muestral. Este análisis arrojó una estructura factorial compuesta por dos factores que en conjunto explicaron el 58,58\% de la varianza, con adecuada confiabilidad interna para el total de la escala $(\alpha=0,816)$ y las subescalas (Tabla 4$)$. Al someter dicha estructura factorial a un análisis confirmatorio se mostró que la estructura propuesta tiene un adecuado ajuste al unir los errores de medición de los reactivos $3 \leftrightarrow 6$ $\left(\mathrm{x}^{2}=14,38 ; \mathrm{CMIN} / \mathrm{DF}=2,055, \mathrm{p}=0,045 ; \mathrm{RMSEA}=0,09 ; 90 \% \mathrm{CI}=0,014\right.$ a 0,166 ; $\mathrm{RMR}=0,065 ; \mathrm{CFI}=0,974 ; \mathrm{TLI}=0,944 ; \mathrm{NFI}=0,952)$ (Figura 1).

Tabla 4. Estructura factorial obtenida en la escala APAIS

\begin{tabular}{|c|c|c|c|}
\hline & \multicolumn{2}{|c|}{ Factor } & \multirow{2}{*}{$\begin{array}{c}\text { Comunalidades } \\
\text { extracción }\end{array}$} \\
\hline & 1 & 2 & \\
\hline \multicolumn{4}{|l|}{ FACTOR 1. Ansiedad por el procedimiento. } \\
\hline \multicolumn{4}{|l|}{$\begin{array}{l}\text { Varianza explicada }=\mathbf{3 6 , 0 5 \%} \\
\text { Alfa de Cronbach }=, 764\end{array}$} \\
\hline APAIS 4. Estoy preocupada por el procedimiento & ,938 & 138 & ,999 \\
\hline $\begin{array}{l}\text { APAIS } 5 . \text { El procedimiento está en mi mente } \\
\text { constantemente }\end{array}$ & ,681 & ,308 &, 559 \\
\hline $\begin{array}{l}\text { APAIS } 6 . \text { Me gustaría saber lo más posible sobre el } \\
\text { procedimiento }\end{array}$ & ,408 & ,249 &, 229 \\
\hline
\end{tabular}

FACTOR 2. Ansiedad por la anestesia.

Varianza explicada $=\mathbf{2 2 , 5 3 \%}$ Alfa de Cronbach $=, 781$

APAIS 2. La anestesia está en mi mente

, $177 \quad, 842 \quad, 741$ constantemente.

APAIS 1. Estoy preocupada por la anestesia. $\quad$, 305

APAIS 3. Me gustaría saber lo más posible sobre la $\quad$, 232 $\quad$,528 anestesia.

Figura 1. Estructura factorial del examen factorial confirmatorio

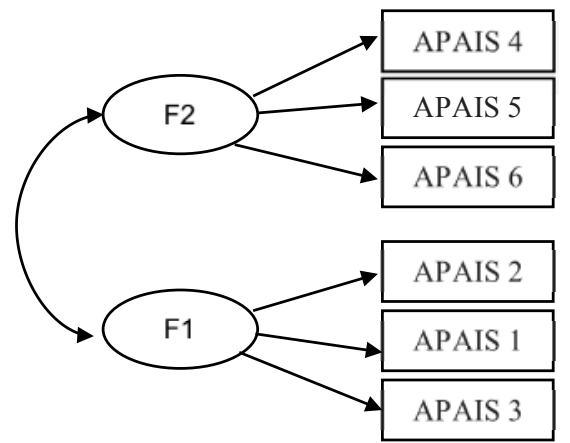


Con el fin de estudiar la validez concurrente del APAIS se estimaron las correlaciones de la escala total y las subescalas con los puntajes obtenidos en el total y subescalas del HADS. Dicho análisis mostró que el APAIS tiene adecuada validez convergente y discriminante al mostrar correlaciones moderadas con la subescala de ansiedad del HADS y correlaciones bajas con la subescala de depresión del HADS (Tabla 5).

Tabla 5. Validez concurrente de la escala APAIS mediante correlación con escala HADS

\begin{tabular}{|c|c|c|c|c|c|}
\hline & $\begin{array}{c}\text { APAIS } \\
\text { anestesia }\end{array}$ & $\begin{array}{c}\text { APAIS } \\
\text { procedimiento }\end{array}$ & $\begin{array}{c}\text { APAIS } \\
\text { Total }\end{array}$ & $\begin{array}{c}\text { HADS } \\
\text { Ansiedad }\end{array}$ & $\begin{array}{c}\text { HADS } \\
\text { Depresión }\end{array}$ \\
\hline $\begin{array}{l}\text { APAIS } \\
\text { Anestesia }\end{array}$ & 1 & & & & \\
\hline $\begin{array}{l}\text { APAIS } \\
\text { procedimiento }\end{array}$ &, $515^{* *}$ & 1 & & & \\
\hline $\begin{array}{l}\text { APAIS } \\
\text { Total }\end{array}$ &, $874 * *$ &, $867 * *$ & 1 & & \\
\hline $\begin{array}{l}\text { HADS } \\
\text { Ansiedad }\end{array}$ & ,318* &, $627 * *$ &, $545^{* *}$ & 1 & \\
\hline $\begin{array}{l}\text { HADS } \\
\text { Depresión }\end{array}$ & ,188 &, $337 * *$ &, $304 * *$ &, $583 * *$ & 1 \\
\hline $\begin{array}{l}\text { HADS } \\
\text { Total }\end{array}$ &, $306^{*}$ &, $593 * *$ &, $519 * *$ &, $961 * *$ &, $786 * *$ \\
\hline
\end{tabular}

${ }^{*} p<, 05 ; * * p<, 01$

Considerando el puntaje obtenido en la subescala de ansiedad de HADS se estimó la sensibilidad y especificidad de la escala APAIS y se observó que el mejor punto de corte para pacientes oncológicos es de 14 puntos (Tabla 6), resaltando que en todos los casos la sensibilidad mantiene puntajes altos, pero la especificidad es baja. A partir de este puntaje se estableció que de la muestra observada $83(70,94 \%)$ de las participantes puntuaron como caso para ansiedad relacionada a la cirugía $(72,2 \%$ mexicanas y $69,80 \%$ costarricenses). Al comparar a las pacientes mexicanas con las participantes costarricenses se pudo observar que no muestran diferencias en la percepción de ansiedad relacionada con la cirugía (Tabla 7).

Tabla 6. Sensibilidad y especificidad de la escala APAIS con diferentes puntajes

\begin{tabular}{lccccc}
\hline & 11 pts. & 12 pts. & 13 pts. & 14 pts. & 15 pts. \\
\hline Sensibilidad & $100 \%$ & $95,65 \%$ & $95,65 \%$ & $95,65 \%$ & $86,95 \%$ \\
Especificidad & $17,95 \%$ & $30,76 \%$ & $38,46 \%$ & $43,59 \%$ & $46,15 \%$ \\
Valor predictivo positivo & $41,81 \%$ & $44,90 \%$ & $47,82 \%$ & $50 \%$ & $51,16 \%$ \\
Valor predictivo negativo & $100 \%$ & $92,31 \%$ & $93,75 \%$ & $94,44 \%$ & $85,71 \%$ \\
\hline
\end{tabular}


Tabla 7. Diferencias en ansiedad prequirúrgica entra participantes de México y Costa Rica

\begin{tabular}{lcccrr}
\hline & $\begin{array}{c}\text { Rango } \\
\text { instrumento }\end{array}$ & $\begin{array}{c}\text { México } \\
\text { M (d. e) }\end{array}$ & $\begin{array}{c}\text { Costa Rica } \\
\text { M (d. e. })\end{array}$ & $t$ & $p$ \\
\hline APAIS anestesia & $3-15$ & $7,87(3,21)$ & $7,73(4,05)$ &, 205 &, 838 \\
APAIS procedimiento & $3-15$ & $10,39(3,56)$ & $9,56(3,52)$ & 1,26 &, 211 \\
APAIS total & $6-30$ & $18,26(6,19)$ & $17,29(6,44)$ &, 83 &, 408 \\
\hline
\end{tabular}

Finalmente se contrastaron las propiedades psicométricas observadas en la escala APAIS obtenidas en la muestra oncológica con validaciones previas de esta escala en diversas muestras y se observa estabilidad en las propiedades psicométricas de la escala, aportando evidencia de la validez de constructo a la misma (Tabla 7).

Tabla 8. Validaciones de la Escala APAIS en diversos países y pacientes sometidos a diferentes tipos de cirugía

\begin{tabular}{|c|c|c|c|c|c|}
\hline $\begin{array}{l}\text { País } \\
\text { (cita) }\end{array}$ & $\begin{array}{l}\text { Tamaño } \\
\text { de } \\
\text { muestra }\end{array}$ & $\begin{array}{l}\text { Características } \\
\text { de participantes }\end{array}$ & $\begin{array}{l}\text { Número } \\
\text { de } \\
\text { factores }\end{array}$ & $\begin{array}{c}\text { Consistencia } \\
\text { interna } \\
\text { (Alfa de } \\
\text { Cronbach) }\end{array}$ & $\begin{array}{c}\text { AFE } \\
\text { (Varianza } \\
\text { explicada) }\end{array}$ \\
\hline $\begin{array}{l}\text { Holanda } \\
(29)^{*}\end{array}$ & 320 & $\begin{array}{l}\text { Cirugías, menor, } \\
\text { intermedio y mayor. }\end{array}$ & 2 & $\alpha=, 86$ & $\mathrm{CP}(72 \%)$ \\
\hline $\begin{array}{l}\text { Alemania } \\
\text { (35) }\end{array}$ & 68 & Cirugía Ortopédica & 2 & $\alpha=, 86$ & $\mathrm{CP}(83,5 \%)$ \\
\hline $\begin{array}{l}\text { Japón } \\
(36)\end{array}$ & 126 & Anestesia general & 2 & $\alpha=, 84$ & $\mathrm{CP}(70 \%)$ \\
\hline $\begin{array}{l}\text { Francia } \\
\text { (37) }\end{array}$ & 175 & Cirugía electiva & 2 & $\alpha=, 84$ & $\mathrm{CP}(85 \%)$ \\
\hline $\begin{array}{l}\text { Malasia } \\
\quad(30)\end{array}$ & 200 & $\begin{array}{l}\text { Sometidos a anestesia } \\
\text { general. }\end{array}$ & 2 & $\alpha=, 90$ & $\mathrm{CP}(79 \%)$ \\
\hline $\begin{array}{l}\text { México - } \\
\text { Costa Rica }\end{array}$ & 117 & $\begin{array}{l}\text { Mujeres con cáncer de } \\
\text { mama }\end{array}$ & 2 & $\alpha=, 82$ & $\begin{array}{c}\text { MV } \\
(58,58 \%)\end{array}$ \\
\hline
\end{tabular}

*Escala original; $\mathrm{AFE}=$ Análisis factorial exploratorio; $\mathrm{MV}=$ Máxima verosimilitud;

$\mathrm{CP}=$ Componentes principales

\section{Discusión y conclusiones}

El objetivo de este estudio fue validar la APAIS en población latinoamericana con cáncer; los análisis realizados mostraron consistencia con validaciones previas en las que se observan dos factores ${ }^{(29-30,35-37)}$ en contraste con la versión original que propone tres factores ${ }^{(29)}$; en las que se pierde como factor la necesidad de información; sin embargo, no se pierden los reactivos, sino que se agrupan a la sintomatología correspondiente. 
La estructura de la APAIS con dos factores muestra consistentemente adecuada validez a lo largo de procesos de validación en múltiples poblaciones y ser sometida a múltiples análisis psicométricos para garantizar su utilidad; dichos hallazgos muestran la estabilidad de la capacidad de la prueba para la evaluación de ansiedad relacionada con la cirugía; lo que da soporte a la validez empírica al instrumento. En cuanto a la integración de los reactivos de información en los dos factores relacionados con el procedimiento y la cirugía; se pueden relacionar debido a factores conceptuales como que, de acuerdo con la Asociación Americana de Psicología (APA), la ansiedad se caracteriza por síntomas fisiológicos y emocionales, pero tiene un componente muy importante sobre la presencia recurrente de pensamientos intrusivos o preocupaciones ${ }^{(39)}$.

La APAIS se centra justo sobre el componente cognitivo de la ansiedad, por lo que la integración de los reactivos de necesidad de información con los factores correspondientes a su sintomatología puede explicarse parcialmente por la alta relación que tiene contar con información precisa sobre el procedimiento y la enfermedad con el desarrollo e intensidad de preocupaciones sobre cada uno de los componentes de la cirugía ${ }^{(40)}$.

Por otro lado, la APAIS muestra adecuada consistencia interna en la población latinoamericana evaluada; la cual es similar a la identificada en estudios previos que abordaron población primordialmente caucásica; lo que indica que se trata de un instrumento sólido. Sin embargo, se observó que el puntaje de corte ideal es más alto en la población latinoamericana con cáncer, lo cual puede estar relacionado con que se trata de un grupo poblacional que al enfrentarse a múltiples estresores relacionados con la propia enfermedad, tienen a presentar niveles más altos de ansiedad que la población general ${ }^{(41,42)}$ y que es consistente con los hallazgos del presente estudio en el que se identificó que con dicho punto de corte casi el $71 \%$ de las pacientes requeriría de atención psicológica especializada para el manejo de ansiedad relacionada con la cirugía.

De manera complementaria, se realizaron análisis de validez convergente y discriminante que nos permiten observar que la APAIS muestra adecuada capacidad de identificar con precisión la sintomatología de ansiedad sin verse interferida por sintomatología psicológica de depresión que frecuentemente se presenta de manera comórbida a la ansiedad ${ }^{(43)}$ y sobre la cual se debe distinguir con claridad para establecer objetivos de abordaje específicos que mejoren las posibilidades de éxito en el manejo especializado.

Las propiedades psicométricas de la APIAS observadas en el presente estudio y en validaciones previas, permiten asegurar que es un instrumento adecuado para evaluar ansiedad relacionada con la cirugía en diversas poblaciones a las que se adhieren pacientes con cáncer y población latinoamericana, que no había sido considerada en estudios previos; cumpliendo así con la posibilidad de evaluar un constructo muy específico que ha sido normalizado y, probablemente subdiagnosticado y poco atendido.

Entre las limitaciones del presente estudio se debe considerar que se contó con una muestra no probabilística que solo incluyó población femenina, por lo que se dificulta la generalización de resultados a otras poblaciones no incluidas; además de que no los hallazgos sobre su validez discriminante son aún limitados. De esta forma futuros estudios sobre la escala en población oncológica podrían centrarse en evaluar la capacidad de la APAIS para discriminar entre ansiedad rasgo, ansiedad asociada al cáncer en general y el objetivo específico de ansiedad relacionada con la cirugía. 
Apesar de las limitaciones señaladas, el presente estudio aporta evidencia sólida sobre la utilidad de la APAIS como instrumento psicológico para evaluar ansiedad relacionada con la cirugía en general y en pacientes oncológicos en particular; lo que puede realizarse en contextos clínicos y de investigación con el fin de aportar conocimientos objetivos para la comprensión del fenómeno de ansiedad ante procedimientos de esta naturaleza y poder desarrollar abordajes de intervención cada vez más específicos para la atención de objetivos muy particulares como es el caso de la ansiedad relacionada con la cirugía. Lo cual, si bien parece un objetivo muy focalizado, puede impactar de manera significativa en la evolución global de los pacientes oncológicos; debido a su sólida y amplia asociación con variables de resultado ${ }^{(21-24)}$.

\section{Referencias bibliográficas}

1. Robles-Castillo J, Ruvalcaba-Limón E, Maffuz A, Rodríguez-Cuevas S. Cáncer de mama en mujeres mexicanas menores de 40 años. Ginecol Obstet Mex 2011; 79: 482-8.

2. World Health Organization. Latest global cancer data: cancer burder rises to 18.1 million new cases and 9.6 million cancer deaths in 2018. 2018 [Acceso 10 de enero de 2019]. https://www.iarc.fr/

3. Instituto Nacional de Estadística y Geografía. Causas de defunción. 2013. Disponible en: http://www3.inegi.org.mx/

4. World Health Organization. Cancer today. Population fact sheets. 2018 [Acceso 10 de enero de 2019]. Disponible en: http://gco.iarc.fr/

5. Martínez F, Rodríguez-Spiteri N, García M, Zornoza G. Novedades en el tratamiento quirúrgico del cáncer de mama. Rev Med Univ Navarro 2008;52: 51-55

6. American Cancer Society. Mastectomía 2018. [Acceso 10 de enero de 2019]. Disponible en: https://www.cancer.org/

7. Herrera-Gómez A, Ñamendys-Silva SA, Meneses-García A. Manual de Oncología: Procedimientos médico quirúrgicos. 6ta ed. México: McGraw Hill, 2016.

8. Paz-Estrada C, Prego-Beltrán C, Barzaga-Hernández E. Miedo y ansiedad a la anestesia en pacientes sometidos a cirugía. Rev Mex Anest 2006; 29: 159-62

9. Gil FL, Costa G, Pérez FJ, Salamero M, Sánchez N, Sirgo A. Adaptación psicológica y prevalencia de trastornos mentales en pacientes con cáncer. Med Clin 2008; 130: 90-2. doi: $10.1157 / 13115354$

10.Zabora J, BrintzenhofeSzoc K, Curbow B, Hooker C, Piantadosi S. The prevalence of psychological distress by cancer site. Psychooncology 2001; 10(1): 19-28. doi: 10.1002/1099-1611(200101/02)10:1\%3C19::AID-PON501\%3E3.0.CO;2-6

11. Thalén-Lindström A, Larsson G, Glimelius B, Johansson BM. Anxiety and depression in oncology patients: a longitudinal study of a screening, assessment and psychosocial support in intervention. Acta Oncol 2013; 52: 118-27. doi: 10.3109/0284186X.2010.707785

12. Jadoon NA, Munir W, Shahzed MA, Choudhry ZS. Assessment of depression and anxiety in adult cancer outpatients: a cross-sectional study. BMC Cancer 2010; 10:594. doi: $10.1186 / 1471-2407-10-594$

13. Valenzuela-Millán J. Barrera-Serrano JR, Ornelas-Aguirre JM. Ansiedad preoperatoria en procedimientos anestésicos. Cir Cir 2010; 78: 151-6

14. Carapia-Sadurni A, Mejía-Terrazas GE, Nacif-Gobera L, Hernández-Ordóñez N. Efecto de la intervención psicológica sobre la ansiedad preoperatoria. Rev Mex Anestesiol 2011; 34: $260-3$ 
15. Gómez-Carretero P, Monsalve V, Soriano JF, de Andrés J. Alteraciones emocionales y necesidades psicológicas de pacientes en una Unidad de Cuidados Intensivos. Med Intensiva 2007; 31: 318-25.

16. Caumo W, Schmidt AP, Schneider CN, Bergmann J, Iwamoto CW, Adamatti LC, et al. Preoperative predictors of moderate to intense acute postoperative pain in patients undergoing abdominal surgery. Acta Anaesthesiol Scand 2002; 46: 1265-71. doi: 10.1034/j.1399-6576.2002.461015.x

17. Alvarez-Bobadilla GM. Ansiedad: evaluación, diagnóstico e intervención preoperatoria. Rev Mex Anestesiol 2014; 37(Supl. 1): S152-S154

18. Scott A. Managing anxiety in ICU patients: the role of pre-operative information provision. Nurs Crit Care 2004; 9: 72-9. doi: 10.1111/j.1478-5153.2004.00053.x

19. Brown PD, Buckner JC, O'Fallon JR, Iturria NL, Brown CA, O’Neill BP, Scheithauer BW, Dinapoli RP, Arusell RM, Curran WJ, Abrams R, Shaw EG. Effects of radiotherapy on cognitive function in patients with low-grade glioma measured by the folstein minimental state examination. J Clin Oncol 2003; 21: 2519-24. doi: 10.1200/JCO.2003.04.172

20. De la Paz-Estrada C, Prego-Beltrán C, Barzaga-Hernández E. Miedo y ansiedad a la anestesia en pacientes sometidos a cirugía. Rev Mex Anestesiol. 2006; 29: 159-62

21. Martínez MA, González ML. Anestesia para mastectomía radical en un hospital oncológico. Rev Med IMSS 2003; 41: 127-31.

22. Gordillo F, Arana JM, Mestas L. Tratamiento de la ansiedad en pacientes prequirúrgicos. Rev Clin Med Fam 2011; 4: 228-33. doi: 10.4321/S1699-695X2011000300008

23. Quintero A, Yasno DA, Riveros OL, Castillo J, Borráez BA. Ansiedad en el paciente prequirúrgico: un problema que nos afecta a todos. Rev Colomb Cir 2017; 32: 115-20.

24. Kagan I, Bar-Tal Y. The effect of preoperative uncertainty and anxiety on short-term recovery after elective arthroplasty. J Clin Nurs 2008; 17: 579-83. doi: 10.1111/j.13652702.2007.01968.x

25. Spielberger CD, Gorsuch RL, Lushene RE. Manual for the State-Trait Anxiety Inventory. Estados Unidos: Universidad de Buffalo.

26. Beck AT, Epstein N, Brown G, Steer RA. An inventory for measuring clinical anxiety: psychometric properties. J Consult Clin Psychol 1988; 56: 893-987.

27. Zigmond AS, Snaith RP. The hospital anxiety and depression scale. Acta Psychiatr Scand 1983; 67: 361-70. doi: 10.1111/j.1600-0447.1983.tb09716.x

28. Krohne HW, de Bruin JT, Mohiyeddini C, Breimer N, Schäfer MK. Specific dimensions of anxiety in surgical patients. Development of a questionnaire and empirical results. Psychother Psychosom Med Psychol 2000; 50: 72-80. doi: 10.1055/s-2000-13236

29. Moerman N, van Dam FS, Muler MJ, Oosting H. The Amsterdam Preoperative Anxiety and Information Scale (APAIS). Anesth Analg 1996; 82: 445-51. doi: 10.1213/00000539199603000-00002

30. Mohd F, Lai LL, Loh PS. Validation of the Malay version of the Amsterdam Preoperative Anxiety and Information Scale. Med J Malaysia 2015; 70: 243-8.

31. Nunnally JCB. Teoría psicométrica. 1a ed. México: Trillas, 2013.

32. García S, Sauri SS, Meza DE, Villagómez AJ. Estado de coma y trastornos de la conciencia: una revisión analítica desde un enfoque neurofuncional. Parte II. Rev Esp Med Quir 2013; 18: 142-7.

33. Migliacci ML, Scharovsky D, Gonorazky SE. Deterioro cognitivo leve: características neuropsicológicas de los distintos subtipos. Rev Neuol 2009; 48: 237-41. 
34. Rico J, Restrepo M, Molina M. Adaptación y validación de la escala hospitalaria de ansiedad y depresiñon (HAD) en una muestra de pacientes con cáncer del Instituto Nacional de Cancerología de Colombia. Avances en Medición 2005; 3: 73-86

35. Berth H, Petrowski K, Black F. The Amsterdam Preoperative Anxiety and Information Scale (APAIS) - the first trial of a German version. Psychosoc Med 2007; 20(4): Doc01.

36. Nishimori M, Moerman N, Fukuhara S, van Dam FS, Muller MJ, Hanaoka K, Yamada Y. Translation and validation of the Amsterdam preoperative anxiety and information scale (APAIS) for use in Japan. Qual Life Res 2002; 11: 361-4. doi: 10.1023/A:1015561129899

37. Maurice-Szamburski A, Loundou A, Capdevila X, Bruder N, Auquier P. Validation of the French version of the Amsterdam preoperative anxiety and information scale (APAIS). Health Qual Life Outcomes 2013; 11: 166. doi: 10.1186/1477-7525-11-166

38. Terol-Cantero MC, Cabrera-Perona V, Martin-Aragón M. Revisión de estudios de la Escala de Ansiedad y Depresión Hospitalaria (HAD) en muestras españolas. Anal Psicol 2015; 31: 494-503. doi: 10.6018/analesps.31.2.172701

39. American Psychological Association. Anxiety. 2018. [Acceso 10 de enero de 2019]. Disponible en: www.apa.org/topics/anxiety/

40. Sjöling M, Nordahl G, Olofsson N, Asplund K. The impact of preoperative information on state anxiety, postoperative pain and satisfaction with pain management. Patients Educ Couns 2003; 51: 169-76. doi: 10.1016/S0738-3991(02)00191-X

41. Thalén-Lindström AM, Glimelius BG, Johansson BB. Identification of distress in oncology patients: a comparison of the Hospital Anxiety and Depression Scale and a Thorough Clinical Assessment. Cancer Nurs 2016; 39: E31-E39. doi: 10.1097/ NCC.0000000000000267

42. Thalén-Lindström A, Larsson G, Glimelius B, Johansson B. Anxiety and depression in oncology patients; a longitudinal study of a screening, assessment and psychosocial support intervention. Acta Oncol 2013; 52: 118-27. doi: 10.3109/0284186X.2012.707785

43. Nikbakhsh N, Moudi S, Abbasian S, Khafri S. Prevalence of depression and anxiety among cancer patients. Caspian J Intern Med 2014; 5: 167-70. 